\title{
Etoposide/Ifosfamide/Mesna Regimen
}

National Cancer Institute

\section{Source}

National Cancer Institute. Etoposide/Ifosfamide/Mesna Regimen. NCI Thesaurus. Code C11266.

A chemotherapy regimen consisting of etoposide, ifosfamide and mesna that may be used in the treatment of breast cancer and recurrent sarcomas. 